\title{
«THE YELLOW WALLPAPER»: ALGUNAS CONSIDERACIONES SOBRE EL DOBLE SUBJETIVO FEMENINO
}

\author{
Rosa Bono Velilla \\ Universitat Autònoma de Barcelona \\ rosabv15@gmail.com
}

Recibido: 20-02-2020

Aceptado: 21-04-2021

(c) $\underset{\mathrm{Br}}{(1)}$

RESUMEN

De los abundantes estudios que abordan la contribución al feminismo de «The Yellow Wallpaper»(Charlotte Perkins Gilman, 1892), apenas alguno presta especial atención al tema del doble más allá de su tratamiento clínico de la enajenación. Este artículo plantea algunas reflexiones respecto al tratamiento literario de la identidad femenina mediante el uso del doble. La aproximación de Jourde y Tortonese (1996) permite una distinción esencial por cuanto considera un rasgo de género interesante: el desdoblamiento femenino suele ser externo u objetivo. El doble subjetivo casi nunca está encarnado por mujeres. El recurso del doble subjetivo femenino, apartado del discurso canónico de la exploración del individuo moderno en este y otros textos que aquí se consideran, ha de dedicarse a indagar y construir su propia identidad: su voz no puede hablar por el conjunto de las inquietudes humanas sino solo por la suya en tanto que mujer.

Palabras clave: doble subjetivo femenino; doble objetivo; Charlotte Perkins Gilman; identidad; literatura fantástica. 


\title{
«THE YELLOW WALLPAPER»: NOTES ON THE FEMININE SUBJECTIVE DOUBLE
}

\section{ABStRact}

Of the many studies that address the contribution Charlotte Perkins Gilman's "The Yellow Wallpaper" (1892) made to feminism, hardly any consider the topic of the double beyond its classification as a symptom of mental disorder. This article looks into the treatment of feminine identity through the use of the double. Jourde and Tortonese's approach (1996) allows for an essential distinction by proposing an interesting gender feature: the splitting of feminine characters usually takes place through external or objective Doppelgänger. The subjective double is almost never embodied by women. The feminine subjective double is thus moved away from the canonical discourse on the exploration of modern identity. Therefore, the literary device is charged with the task of examining and producing its own identity: its voice cannot speak for the entirety of human concerns, only for itself as a woman.

KEY-WORDS: Gómez de Avellaneda ; feminine subjective double ; objective double ; Charlotte Perkins Gilman ; identity ; fantastic literature.

\begin{abstract}
$\infty$
Une sorte d'ombre qui me donne à moi-même ma propre visibilité, qui me permet de me regarder là où je suis absent utopie du miroir.
\end{abstract}

M. FoucAulT, «Des espaces autres. Hétérotopies» (1967)

Parece que en los últimos años se ha enmendado con creces la omisión del relato más famoso de Charlotte Perkins Gilman. Hasta la nueva ola de feminismo tras la primera mitad del siglo xx, «The Yellow Wallpaper» (The New England Magazine, enero de 1892) había pasado prácticamente desapercibido tanto para la crítica como para las editoriales y el público lector. Dejó de imprimirse durante cincuenta años, hasta que The Feminist Press retomó su edición en 1973. Mientras los textos no ficcionales de la autora formaban parte del programa didáctico de algunas universidades estadounidenses ya desde los años veinte - aunque también dejarían de imprimirse en las siguientes décadas-, su ficción sería alabada esporádicamente y por escuetas reseñas en 
alguna antología como la de William Dean Howells (Great Modern American Stories), que se redujo a destacar lo escalofriante del relato. Elaine R. Hedges explica esta laguna en uno de los primeros estudios exhaustivos del texto, publicado a modo de epílogo en la reedición de 1973. La autora se propone sumar su interpretación de «The Yellow Wallpaper» al redescubrimiento de la literatura de Gilman: «no one seems to have made the connection between the insanity and the sex, or sexual role, of the victim, no one explored the story's implications for male-female relationships in the nineteenth century» (Hedges, 1988: 41).

A la observación preliminar de Hedges han sucedido numerosos artículos que multiplican los comentarios a la «histeria femenina» que ilustra el relato. Cabe mencionar entre estos la única edición crítica de la obra, a cargo de Julie Bates Dock, un estudio riguroso de la historia textual del relato que discrepa de la dicotomía entre la recepción original y las lecturas críticas posteriores: «contemporary reviews demonstrate that the first readers did recognize its indictments of marriage and of the treatment of women» (1998: 19). Sea desde la psicología, la narratología o el comparatismo, la crítica reciente se ha servido del texto por dos vías fundamentales: la del feminismo, examinando el texto como muestra de la disrupción traumática de la lógica social del patriarcado; y la del historicismo, tomándolo como confirmación de esa dinámica histórica inquebrantable. Conviene aquí, pese a las múltiples perspectivas, tener presente y coincidir en que el relato de Gilman acomete el examen de una condición esencialmente femenina y que el suyo es un litigio acotado por circunstancias históricamente adscritas a la mitad de la población. El anonimato de la protagonista frente al resto de personajes que intervienen en la acción, a los que la autora sí da nombre, no podría ser más significativo como símbolo de esta identificación colectiva.

También a pesar de la profusión bibliográfica, muy raramente se ha abordado la cuestión desde el estudio de un eje de articulación principal en el relato: el tema del doble. ${ }^{1}$ De forma casi exclusiva, la presencia de esa otra mujer en la historia y la transmutación de la protagonista se han tratado en términos de alucinación, esquizofrenia o, en palabras de Gilman, «incipient insanity», «nervous breakdown», «melancholia», «neurasthenia» (1913: 331), ${ }^{2}$

1 Rebeca Martín (2006: 13) trata la complejidad terminológica en la clasificación del ámbito del doble — «tema», «constelación temática», «motivo», «archipiélago»—. La inabarcable variabilidad de los matices asociados a este ámbito suscita una discusión potencialmente infinita que resulta imposible siquiera tantear en un análisis como este, por lo que no suscribiremos de forma estricta una $u$ otra taxonomía.

2 Cfr. Quawas (2006), y Santana (2018). 
lo cual en absoluto es desacertado si ha de entenderse, como hacen Hedges (1988) y Dock (1998), que son esos términos los que levantan cargos contra la condición de la mujer decimonónica. Sin embargo, no es menos cierto que las posibilidades de canalización de un motivo como la locura son tan vastas cuanto significativa la precisión de optar por el enajenamiento de la dualidad para explorar el extrañamiento de la propia imagen, una inquietud bien asentada en el interés de la cultura occidental por esta y otras patologías desde el siglo XIX (Fusillo, 1998: 266). Hasta donde sabemos, solo una de las excepciones profundiza razonablemente en este aspecto: Margaret Delashmit, que estudia la representación del doble «as evil self» en este relato y otros a partir de las teorías de Rank (1971) y Freud (1957), concibe el patrón del empapelado de donde emerge la segunda mujer como imagen de la lógica paternalista masculina que atrapa a la narradora:

tension results (...) when the ego opposes the id or the superego opposes the ego, for example. Let repression become too severe, and the personality may divide and the divided part then may project itself onto some «real or imagined other» (...) The narrator in Gilman's story, therefore, projected her identity upon the woman in the wallpaper because that identity, though it resulted in insanity, presented a lesser danger to her sense of self than did living in John's world (1990: 150).

Se trata de una vinculación frecuente en la crítica del psicoanálisis entre la duplicación y el síntoma clínico: «The Doppelgänger may be said to act as the vicarious agent of repressed fantasies, where repression renders the fantasy hysterical or paranoid. The Doppelgänger story is thus recurrently marked by compulsive repetitions, hysterical crises, and the obsessive terror of persecution fantasies» (Webber, 1996: 17). Las fantasías recurrentes de la protagonista (la palabra «fancy» es de las que más se repiten en el texto) derivan precisamente en la histeria del estampado múltiple y la identidad reiterada, y de ahí en la emancipación y la agonía que cierra el relato. Coates (1988: 104) acude a Masse und Macht de Elias Canetti (1960) para asociar la histeria a la transformación violenta como vía de escape del personaje cautivo. Tal podría ser el caso de la narradora de Gilman.

Los análisis fisiologistas del doble convienen perfectamente a la trama de «The Yellow Wallpaper», pero insisten más en el desdoblamiento como forma de alienación psicológica que en su relevancia como efigie de la liberación feminista, un efecto ulterior solo visible desde una perspectiva de conjunto del doble femenino. Así, una lectura biográfica, acorde úni- 
camente a lo que la autora declaró de forma explícita sobre su relato, puede llegar a eclipsar la trascendencia de la duplicidad de la protagonista, la cual, por otro lado, en modo alguno debió de ser extraña al propósito de Gilman.

Una vez apreciada la importancia del tema del doble en «The Yellow Wallpaper», es preciso reparar en que casi ninguna teoría permite explorar o tiene en cuenta siquiera la particularidad del elemento femenino en la configuración del tema. Las distinciones de género son poco habituales a la hora de abstraer y clasificar las nociones que determinan los tipos de Doppelgänger. En la crítica literaria del doble, el estudio del cuestionamiento y disolución de nociones como consciencia, unidad o diferencia — que caracterizan el temasuele contemplar la condición humana como entidad homogénea, como una sola, de validez universal, representada por un «sujeto moderno» que figura inquietudes compartidas por todos. El género no marcado es aquí también el masculino. La mayoría de los casos entraña y valida en efecto esa uniformidad, pero ¿qué hay de las ficciones del doble cuyos interrogantes apelan únicamente a la otra mitad de la población? ${ }^{3}$ Taxonomías estructuralistas como la de Doležel (2003), aunque admiten variantes, no ofrecen herramientas para integrarlas y delimitan las características del tema con una rigidez que quiebra sus propias bases al mínimo desajuste en la práctica. No obstante, la mayoría de propuestas teóricas son flexibles y parten de la variación para acotar el doble literario, por lo que podrían permitir fácilmente los matices de una línea temática como la sugerida, por ejemplo el enfoque inductivo y diacrónico del campo temático de Fusillo (1998), que retoma la distinción tema-concepto de Bremond (2003); la «constelación temática» de Vilella (1999), o el desgaje de elementos simples que permiten tipologías dinámicas, de Jourde y Tortonese (1996).

La exégesis marxista que plantea Todd McGowan para «The Yellow Wallpaper», si bien aspira a indagar el carácter del desafío femenino en el texto, resta trascendencia a esta particularidad: la mujer que narra la historia es cosificada por el capitalismo como el resto de los seres humanos, por lo que su actitud simboliza una condición humana general, una idea que, como veremos, no es consecuente con los usos literarios de la época. La desposesión material conduce a la consciencia del vacío de la propia identidad (hu-

3 Existen estudios críticos sobre el doble femenino aplicados a obras concretas, como The Female Fantastic (2009), de Gloria Alpini, que dedica un capítulo al estudio de la figura del doble en la escritura de Ada Negri, tomándola como ejemplo de la constitución de la identidad femenina a través de su erosión mediante el recurso narrativo del Doppelgänger (2009: 113). 
mana) y así a la liberación, que, paradójicamente, es facilitada por la sumisión patriarcal:

economic factors - and John's patriarchal authority that invokes them - force, or make possible, the narrator's following the path of her desire. (...) The power of John to compel the narrator to remain in a situation that constantly horrifies her-the power of patriarchy itself-is simultaneously the impetus for her attempt to free her self [sic] from the wall-paper (McGowan, 2001: 43).

Con todo, la idea de reificación pone de relieve un aspecto fundamental en el tratamiento del motivo del doble femenino, ya que esta variante queda relegada por defecto a la manifestación objetiva o externa del Doppelgänger. Sin percibir la parcialidad del recurso, McGowan no advierte que el cuestionamiento de la identidad humana, por defecto, no se lleva a cabo desde el desdoblamiento femenino, que este ha de ocuparse primero en explorar y afirmar o rechazar su lugar en el mundo en tanto que femenino antes que humano. Al analizar las categorías de género como construcción ideológica, Judith Butler se pregunta: «What sense does it make to extend representation to subjects who are constructed through the exclusion of those who fail to conform to unspoken normative requirements of the subject?» (1990: 9). El relato de Gilman constituye una excepción interesante justamente por incluir en la representación del sujeto a ese sector que no satisface sus moldes normativos. Webber (1996: 17) analiza otra forma de heterodoxia en la literatura del doble que parte, precisamente, de casos de "“female complaint" of hysteria» en sujetos masculinos como los que plantean Jekyll y Hyde o el estudiante Törless, de Robert Musil. Otro ejemplo en nuestra literatura sería el de «La Borgoñona», de Emilia Pardo Bazán, donde el doble objetivo es masculino. Si bien, como indica Webber, estos casos subvierten o problematizan nociones de género en el tema del doble, es innegable que lo predominante, como él mismo reconoce, es el tratamiento de lo femenino en «lo otro», mientras que el sujeto-Doppelgänger «often figures as an agent of free-wheeling virility» (1996: 17). Rebeca Martín señala la representación descompensada del doble en la literatura hasta el siglo xx:

En la literatura del siglo xIx el doble es, en efecto, generalmente masculino (...) La literatura fantástica decimonónica está en su mayoría escrita y protagonizada por hombres, mientras que los personajes femeninos desempeñan un papel secundario. El caso del doble, lejos de ser una excepción, supone incluso un exacerbamiento de la norma: el fenómeno autoscópico pone en cuestión, ante todo, la identidad del original, y no debe olvidarse que, oficialmente, esta se concebía en términos masculinos. El hecho sobrenatural amenaza aquello que de algún modo 
está establecido, sancionado por la convención, la lógica o la moral pública, y la identidad de la mujer constituía todavía un ente en construcción (2006: 48-49).

Gilman advirtió tal falta en la sociedad contemporánea y la denunció en unos términos análogos que disociaban feminidad y humanidad:

the house-bound woman is (...) denied time, place, and opportunity to develop those characteristics. She is feminine, more than enough, as man is masculine more than enough; but she is not human as he is human. The house-life does not bring out our humanness, for all the distinctive lines of human progress lie outside (Gilman, 1910: s.p.).

La aseveración de la autora respecto a la reclusión doméstica de la mujer no podría ser más cabal en relación con la presencia femenina en la literatura del doble: es femenina, ante todo, pero ha de serlo necesariamente porque se le ha negado el espacio de representación artística que podría considerarla «humana». ${ }^{4}$ Por lo demás, lo convencional y arraigado de la situación en la época bien puede sugerir reformular el sujeto en un «yesterday's and today's woman». Fuese o no su propósito sentar precedente y llamar la atención sobre el hecho de que el recurso del doble no tuviese ministerio entre las mujeres, lo cierto es que, aún en las postrimerías del siglo XIX, Charlotte Perkins Gilman, en contra de la corriente genérica masculina, se vale del doble femenino para explorar ese «ente en construcción» que era la mujer.

La distinción de Jourde y Tortonese entre doble subjetivo y doble objetivo (1996: 92) ${ }^{5}$ es la más conveniente para expresar la dicotomía del género en el tema del doble, principalmente porque considera un factor interesante al respecto en la caracterización del segundo. A menudo, el doble objetivo -

4 La teórica feminista Teresa de Lauretis precisa que además es necesario cambiar los paradigmas de representación de los modelos femeninos dentro de ese espacio. Su monografía Alicia ya no (1992) estudia la semiótica cinematográfica asociada a la imagen de la mujer a partir del psicoanálisis, eminentemente desde la sexualidad. Lauretis (1992: 74) contempla el dinamismo de las representaciones icónicas —femeninas—, su historicidad ligada a la recepción frente a la rigidez de los sistemas semióticos preestablecidos (formalistas) que limitan dichos paradigmas. La incursión del doble subjetivo femenino en la predominancia de los modelos objetivos y / o masculinos del doble podría asociarse al proceso que presenta Lauretis.

5 La «reificación» que aquí hemos vinculado a la manifestación objetiva del doble femenino puede resultar confusa si se considera la idea de «réification du sujet» que utilizan Jourde y Tortonese (1996: 92) para explicar el doble subjetivo. Sin embargo, las nociones no son excluyentes: el significado de la primera es plenamente intuitivo y etimológico — tanto Jourde y Tortonese (1996: 101) como Martín (2006: 350) recurren a la ambivalencia del término «objeto» para caracterizar al doble objetivo femenino-, mientras que en el segundo caso denota la escisión que pone al personaje enfrente de una réplica suya que automáticamente lo hace externo a sí mismo, es decir, objetivo o reificado (de ahí el conflicto que origina respecto al límite entre lo propio y lo ajeno). 
aquel por el que el desdoblamiento se da en un ente externo al protagonista, que a menudo es también narrador- aparece formulado en la trama como reaparición de la mujer amada. ${ }^{6}$ La relación entre los dobles objetivos, apuntan los autores, refiriéndose particularmente al doble femenino, es «une différence sans contenu, une différence vide: la seconde est exactement la même que la première» (1996: 102). No existe diferencia entre una mujer y otra precisamente a propósito de su reificación: lo verdaderamente inquietante de la repetición de un objeto o un ser externo a nosotros es la exactitud de la copia, tan insólita en la naturaleza; así como el parecido gemelar nos asombra más cuanto más afines son los hermanos. La clasificación subjetivo-objetivo separa el cuestionamiento de la identidad del cuestionamiento de las leyes del mundo. Hay una progresión inversa: mientras el doble del personaje principal —o subjetivo- parte del principio de unidad para derivar en la disgregación de la propia existencia y el abismo de la incoherencia, el doble exterior va de la presunción de diferencia al énfasis en lo problemático de una paridad exacta. ${ }^{7}$ La disgregación del sujeto contribuye en última instancia a su crisis o a su creación, mientras que la reiteración del objeto no suele ir más allá de una disposición escéptica y aun de la repulsa hacia lo incomprendido. La mujer, en su reducción al papel de doble objetivo, no participa de la exploración de la problemática humana ni la significa, sino que queda relegada al ámbito de lo material e inaccesible. En este sentido, la representación femenina, como la del objeto reiterado espontáneamente, no da pie más que a la turbación que despierta lo preternatural.

Así, el concepto de lo subjetivo en el tema del doble establece un límite esencial para distinguir en la práctica literaria las dos grandes inquietudes de la modernidad - la realidad y el yo- y es a la vez lo suficientemente amplio para integrar cualquier interrogante acerca de la identidad que pueda suscitar la duplicación. Por ejemplo: ¿qué es de lo femenino en el ámbito del doble subjetivo? La ausencia de que venimos hablando no es exclusiva del Doppelgänger - ya apunta Martín que se extiende a la literatura fantástica - pero tiene especial

6 Algunos ejemplos: Aura, de Carlos Fuentes; Fort comme la mort, de Guy de Maupassant; Bruges-la-Morte, de Georges Rodenbach; o «El desertor», de José María Merino.

7 Valga como ejemplo del proceso de extrañamiento subjetivo el clásico «William Wilson» de Poe. La inquietud y la enajenación del narrador aumentan al mismo ritmo que el parecido entre el protagonista y su doble: «The feeling of vexation thus engendered grew stronger with every circumstance tending to show resemblance, moral or physical, between my rival and myself» (Poe, 2009: 763). En cuanto a la dinámica de extrañamiento respecto al doble objetivo, la disrupción afecta a la realidad externa y sus principios lógicos antes que a la identidad de la voz narrativa. La confirmación de ese tipo de disrupciones constituye la anagnórisis en relatos como Aura, donde la -imposible pero cierta-identidad entre la joven sobrina y su anciana tía se revela a partir de las fotografías que ve Montero en el último capítulo. 
sentido estudiarla aquí por su función deconstructiva y regeneradora, por el tipo de indagación sobre el sujeto moderno que permite el tema del doble. Hemos empezado considerando «The Yellow Wallpaper» por cuanto supone un precedente y una excepción clara que permite trazar un hilo de continuidad durante el siglo siguiente, una constante que puede hacernos cuestionar si ha habido algún avance efectivo hacia la integración de la mujer en el repertorio universal de la identidad humana disgregada. ${ }^{8}$ Para ver el alcance de cualquier hipótesis al respecto hay que tomar perspectiva y contemplar el relato de Charlotte Perkins Gilman a la par de otros textos. Tomaremos aquí otros tres casos que se ajustan a variantes diacrónicas y diatópicas, con el fin de observar cómo varía el motivo de la voz femenina como forma de desafío del discurso patriarcal a través del doble subjetivo. Por un lado, un poema contemporáneo a la narración de Gilman, «The Other Side of a Mirror» (1896), de Mary Elizabeth Coleridge. ${ }^{9}$ Por otro, dos cuentos: uno medio siglo

8 Aunque el tema del doble femenino no ha recibido una atención homogénea ni suficiente por parte de la crítica, los estudios del feminismo y la otredad llevan décadas explorando la construcción de la feminidad a través de las pautas de género y sexualidad establecidas implícitamente por la escritura, también desde lo fantástico. Véase, al respecto, la monografía de Gloria Alpini, The Female Fantastic. Evolution, Theories and the Poetics of Perversion (2009), y las antologías de Gayle Greene y Coppelia Kahn, Making a Difference: Feminist Literary Criticism (1985), y de Elizabeth Abel, Writing and Sexual Difference (1982), además del conocido libro de Judith Butler, Gender Trouble: Feminism and the Subversion of Identity (1990).

9 La crítica tradicional, encabezada por teóricos como Roger Caillois en Au coeur du fantastique (1965) y Tzvetan Todorov en Introduction à la littérature fantastique (1970), descarta la lírica como vehículo expresivo de lo fantástico: para que pueda considerarse la fantasticidad de un texto, este debe enunciar la ficción en sentido literal, es decir, ha de expresar de forma representativa el componente sobrenatural que lo define (Todorov, 1994: 55). El carácter referencial u objetivo de la narración propicia esta condición mejor que ninguna otra modalidad discursiva, especialmente si se compara con la subjetividad expresiva asociada a la poesía lírica desde el Romanticismo. Recientemente María E. Llorente (2019) ha defendido la posibilidad de contar la lírica entre las manifestaciones de lo fantástico, partiendo de las propuestas que no tardaron en oponerse a aquellas primeras teorías. Dichas propuestas — definidas, entre otros, por Bárbara Smith, en «La poesía como ficción» (1971); Susana Reisz, en «Ficcionalidad, referencia, tipos de ficción literaria» (1979); José Ma Pozuelo Yvancos, en «Lírica y ficción» (1991); y Eduardo García, en Una poética del límite (2005) — admiten la capacidad ficcional y referencial de la poesía lírica, y así su aptitud para verbalizar lo fantástico. Mediante la reinterpretación de los principios todorovianos, Llorente (2019: 310) propone tres condiciones para la lírica fantástica: «1) la afirmación de lo enunciado por el hablante como verdad textual; 2) la posibilidad de que esta afirmación tenga también, en un segundo plano, un sentido alegórico o metafórico, que representa o describe el estado emocional y anímico del hablante; y 3) la presencia y distribución de lo fantástico de manera homogénea y el mantenimiento de un mismo tono a lo largo de todo el texto». Puede considerarse que el poema de Mary E. Coleridge cumple esos tres principios: el primero, mediante la modalidad afirmativa en primera persona, análoga al narrador omnisciente, y el modo indicativo; el segundo, meramente admitiendo la compatibilidad entre la interpretación figurada del texto (la mujer del espejo es una representación hiperbólica del estado de ánimo de la protagonista) y una lectura literal (la imagen femenina en el espejo es otra mujer, a la que se alude en tercera persona y no en primera, a diferencia de la voz que enuncia); el tercero, advirtiendo cómo cada verso sostiene el horror de la alienación y notando la traslación que propone el título al evocar el «otro lado del espejo» (inexistente en realidad: sobrenatural) como espacio tangible donde la mujer aparecida escucha hablar a la protagonista («The ghost of a distracted hour, / that heard me whisper»; Anodos, 1896: 11). A estos argumentos puede añadirse que el canon inserta el poema en la literatura gótica, una de las vetas más fecundas de lo fantástico. 
posterior, «Lejana» (1951), de Julio Cortázar; y, ya a finales del siglo xx, «Lúnula y Violeta» (1980), de Cristina Fernández Cubas. La constante mencionada aparece de una forma $\mathrm{u}$ otra en los cuatro textos, que este artículo no aspira a analizar pormenorizadamente sino por cuanto ofrecen ejemplos, matices y nuevas perspectivas en la evolución del recurso estudiado. ${ }^{10}$ Los relatos recuperan la cuestión crónica de la actividad literaria femenina, y cada uno de los textos revela a su modo una preocupación por la voz del personaje principal que siempre revela una voluntad de expresión frustrada. Se trata de una dimensión metaliteraria que no es inherente a la literatura del doble, ni mayoritaria, pero en la que la variante estudiada insiste notablemente (también aparece, por ejemplo, en La soledad era esto [1990], de Juan José Millás). Un enfoque comparativo permitirá considerar, finalmente, si la figuración femenina como doble subjetivo sigue dedicándose a explorar y construir su identidad como mujer después del XIX, o si por el contrario ha logrado sumarse al elenco de la representación de las inquietudes humanas.

En nuestro texto de referencia principal son constantes las alusiones de la protagonista a los obstáculos que encuentra para escribir, trabas que son en principio externas, impuestas por el marido y vigiladas por la hermana, pero que llegan a hacer que ella misma cuestione la conveniencia de lo que concibe como su «trabajo»:

So I take phosphates and phosphites (...) and I am absolutely forbidden to «work» until I am well again. (...) I did write for a while in spite of them; but it does exhaust me a good deal-having to be so sly about it, or else meet with heavy opposition. (...) I think sometimes that if I were only well enough to write a little it would relieve the press of ideas and rest me. (...) I don't know why I should write this. I don't want to. I don't feel able (Gilman, 1973: 10, 16, 21).

10 Se recurre aquí al término «voz» porque el motivo de la escritura no aparece en el poema de Coleridge, pero ambas variantes significan por igual la expresión de las protagonistas, que es donde en última instancia reside el sentido de la manifestación de la feminidad. Otro motivo que se reitera en tres de los textos seleccionados es el del reflejo (en el nombre de Lúnula, en el estampado de la superficie del empapelado y en el espejo del poema). Si bien en general «ombres, reflets, portraits réifient le sujet, l'inscribent dans le monde des choses» (Jourde y Tortonese, 1996: 93), — pensemos en Dorian Gray, en «Diálogo en el espejo» de García Marquez, en «Dos imágenes en un estanque» de Giovanni Papini o en «The Shadow» de Hans Christian Andersen, entre muchos otros- la imagen ha adquirido para la crítica connotaciones específicas cuando es una mujer la que se encuentra frente al espejo: «could it be that woman is so often represented as seated in front of a mirror because she seeks thereby to come to terms with the disparity between her mental self-image, an imaginary form contaminated by the prevailing male images of the patriarchal culture, and the actual fact of her difference?» (Coates, 1988: 4). La reflexión tiene especial pertinencia en el caso de «Lúnula y Violeta», donde el reflejo tenuemente masculinizado de la protagonista, si bien inicialmente la ayuda a dejar de preocuparse por su «aborrecida imagen», acabará contaminándola hasta suplantarla por completo y destruirla. 
La represión de todo estímulo intelectual y físico provoca una angustia que se focaliza en el estampado del papel amarillo, el cual termina siendo estudiado con una precisión enfermiza por la narradora. Finalmente, una figura femenina emerge de la pared y, por momentos, se multiplica en otras mujeres que no hacen más que arrastrarse continuamente. La configuración del doble es ciertamente peculiar, y casi podría descartarse de no ser por la transfiguración final de la protagonista mediante la voz narrativa, que pasa de identificar a la mujer de la pared en tercera persona a darle expresión en primera. La metamorfosis que idea Gilman es brillante: la histeria actúa como catalizador de la transformación de una mujer que se expresa, pero no logra sobreponerse al dogma (escribe a escondidas), en otra que no habla pero que, literalmente, se atreve a pasar por encima del hombre desfallecido una y otra vez. Es entonces, con la fusión en una misma mujer, cuando la primera puede seguir escribiendo, por un momento al menos, y la segunda recibe voz: «Now why should that man have fainted? But he did, and right across my path by the wall, so that I had to creep over him every time!» (1973: 36). De esta forma, la acción realmente asertiva que perseguía Gilman ${ }^{11}$ se ha logrado a través del doble por fusión, según los tipos que propone Bargalló (1994). ${ }^{12}$

Los otros tres textos presentan concomitancias respecto al motivo de la voz/escritura más o menos afines al primer relato. En el poema de Mary E. Coleridge, la voz poética conjura el reflejo de una mujer cuya apariencia difiere del ideal femenino de la época victoriana:

The vision of a woman, wild

With more than womanly despair.

Her hair stood back on either side

A face bereft of loveliness.

(...)

Her lips were open - not a sound

Came though the parted lines of red

(...)

No sigh relieved her speechless woe,

She had no voice to speak her dread.

(...)

11 «I try to describe this long limitation, hoping that with such power as is now mine, and such use of language as is within that power, this will convince any one [sic] who cares about it that this "living" of mine had been done under a heavy handicap» (Gilman, 1990: 104).

12 La clasificación de Bargalló consta de tres procedimientos de duplicidad: por fusión (acercamiento progresivo de los dobles hasta su identificación, como en el relato de Gilman), por fisión (desdoblamiento del personaje, como ocurre en Nos, de Gogol) y por metamorfosis (transformación, con el ejemplo clásico de Dr. Jekyll and Mr. Hyde, de Stevenson). 
The ghost of a distracted hour,

That heard me whisper: - «I am she!» (Anodos, 1896: 10-11).

De nuevo, la expresión se consigue a través del desdoblamiento y con el paso de la primera persona a la tercera en referencia a la mujer del espejo, así como con la identificación final con un doble mudo y en un principio amenazante. Heather Braun se ocupa del doble y de las voces fragmentadas en la producción de la autora, de cómo estas sirven para poner en entredicho los presupuestos sociales victorianos respecto a la mujer:

More importantly, the fragmented, epicene speakers (...) complicate rigid thinking about Victorian women as passive, dutiful, and angelic mothers and wives. (...) Coleridge's androgynous, often duplicitous narrators challenge the notion that literary Doppelgängers embody secrets hidden deep within a male psyche. Rather, her work actively rejects the belief that Victorian women were incapable of compelling duplicities all their own (2016).

Muy cercana en el tiempo a Gilman, la obra de Mary E. Coleridge recurre al doble subjetivo femenino como resorte simbólico de la voz usurpada y sustituida por un discurso ajeno, incapaz de representar o acoger cualquier tipo de feminidad que no haya promulgado él mismo, hasta que la propia protagonista se reconoce en esa proyección y la utiliza para definirse. De este modo, su poesía proyecta duplicidades «all their own», voces propiamente femeninas que desafían abiertamente los modelos de la pasividad y del silencio de la mujer en la literatura.

«Lejana» es el único texto en el que, aparentemente, el tema de la escritura no se vincula de forma directa con la presencia del doble, pero la situación que describe la voz narrativa del cuento de Cortázar es, medio siglo más tarde, la misma que la referida por Gilman: «Y sin embargo, ya que cerraré este diario, porque una o se casa o escribe un diario, las dos cosas no marchan juntas (...) (Lo escribo, y basta de diario para bien mío.)» (1982: 47). Hay, incluso, un indicio de enfermedad que podría sugerir la enajenación, cuando Alina empieza la entrada de sus memorias el 7 de febrero: «A curarse» (1982: 23). Esta vez, el intercambio de identidades supondrá un salto a la tercera persona, un distanciamiento, y dos meses después, el divorcio, porque Alina ya no es la misma mujer; no sabemos si la nueva Alina ha transgredido el único criterio mencionado expresamente por la narradora para un matrimonio lícito y para bien suyo: no escribir.

«Lúnula y Violeta», sin embargo, se construye entorno a la escritura como tema principal. Si en el poema de Coleridge la expresión emerge en el 
último verso con una exclamación casi triunfal, la voz de Lúnula va supliendo la escritura de su amiga progresiva y violentamente: «A medida que avanzo en la lectura veo que el lápiz, tímido y respetuoso, ha sido sustituido por una agresiva tinta roja. En algunos puntos apenas puedo reconocer lo que yo había escrito. En otros tal operación es sencillamente imposible: mis párrafos han sido tachados y destruidos» (Fernández Cubas, 1988: 20). Podríamos contentarnos simplemente con señalar la anomalía y entender, como hace Anne Poelen (2005: 245), que es una mujer quien suplanta la identidad de la protagonista, pero algún pasaje del texto —además del carácter campesino y las actitudes rudas de Lúnula, típicamente masculinas en el imaginario de la cultura occidental - nos hace estar alerta ante sutiles intrusiones del componente de dominio masculino: «Recuerdo nuestra visita al viejo almacén e imagino nuestro aspecto en el café: una mujer sentada junto a un bulto del que, a primera vista, resultaba difícil distinguir el sexo» (Fernández Cubas, 1988: 21). El tipo de violencia que en «Lejana» es tan agresivo por ser directo (el verbo «pegar» se repite una y otra vez) se ha convertido en la famosa «toxicidad», en un acoso apenas identificable, desconcertante justamente por sus implicaciones de género.

A lo largo de un siglo, cuatro textos pertenecientes a cuatro tradiciones literarias distintas mantienen el motivo de la «Voz» de la protagonista como contrapartida de los términos canónicos que imponen una definición por ausencia. Es una continuidad que, como mínimo, nos habla de una condición todavía vigente. Nos haremos eco de la pregunta de Sandra Gilbert y Susan Gubar en uno de sus estudios sobre la historia de la escritura femenina para entender la trascendencia de la cuestión:

What does it mean to be a woman writer in a culture whose fundamental definitions of literary authority are, as we have seen, both overtly and covertly patriarchal? (...) the woman writer's male precursors symbolize authority; (...) they fail to define the ways in which she experiences her own identity as a writer. More, the masculine authority with which they construct their literary personae, as well as the fierce power struggles in which they engage in their efforts of self-creation, seem to the woman writer directly to contradict the terms of her own gender definition (1979: 45-48).

Las formas tradicionales de la autoridad literaria no logran dar cuenta de la identidad femenina, por lo que esta ha de procurarse sus propios medios de representación (no entraremos en cuestiones de autoría, pero, como demuestran estas lecturas, no se trata de un desagravio unilateral). En tanto que forma 
privilegiada de cuestionamiento y construcción de la identidad, el tema del doble resulta especialmente oportuno para abordar la escritura como parte fundamental de esa definición exclusivamente femenina. La condición general que exponen Gilbert y Gubar puede aplicarse aquí de forma bien específica por cuanto la vertiente tradicional del tema ha excluido sistemáticamente la representación literaria de la mujer como sujeto en sus obras, por lo que esta ha de buscar su propia voz, que puede encontrar en el desdoblamiento, como sucede en el poema de Mary E. Coleridge. Una vez encontrada esa voz, y liberada, la escritura puede ser el medio para equiparar los sexos, como lo fue para Gilman, y lograr lo humano también desde lo femenino:

Literature was a great world-art for centuries and centuries before women were allowed to read-to say nothing of write! (...) In our last century, in civilised Christian England, Harriet Martineau and Jane Austen covered their writing with their sewing when visitors came in; writing was «unwomanly!» (...) A larger range of interest and care instantly gives a resultant largeness of mind, in women as well as men. Such free great lives as have been here and there attained by women show the same broad human characteristics as similar lives of men (Gilman, 1910: s.p.).

De nuevo, el efecto metaliterario de la otredad de la mujer que escribe ofrece vías de regeneración particularmente convenientes a ese proceso de apertura y asimilación. Ahora bien, el optimismo de Gilman, compartido por Gilbert y Gubar (1979: 49) al hablar de una circunstancia extraliteraria en vías de solución, no es del todo consecuente con lo que propone la mayoría de los ejemplos aducidos como muestra representativa. Por mucho que lo ominoso sea característico de la literatura del doble, no dejaremos de preguntarnos qué sentido tiene en los tres relatos a los que nos hemos asomado: la ficción configura en términos ambiguos la consecución de esa voz que venimos reivindicando y del equilibrio («same broad human characteristics») al que aspira en último término. Paradójicamente, la sola existencia de estos casos de doble subjetivo femenino prueba alguna efectividad al respecto, pero su misma vigencia niega que haya habido una integración efectiva de la mujer en lo humano-masculino del discurso literario canónico. Seguramente, como sugiere Butler (1990: 6), la representación del sujeto femenino solo tendrá sentido cuando la particularidad de la mujer como sujeto deje de presumirse, es decir, cuando esa presencia pueda indicar, como cualquier otra, la identidad humana sin marca de género significativa. La continuidad que evidencian estos ejemplos, ¿es muestra de rupturas sucesivas que quiebran y derriban una lógica de representación domi- 
nante fundada en lo masculino-humano? Si, como creía Dock (1998: 19) en referencia a «The Yellow Wallpaper», cada sociedad reconoce las acusaciones de sus textos y todavía es necesario que exista un doble subjetivo femenino desde el que problematizar únicamente la feminidad, ¿será que solo ha cambiado la sutileza con que se relega la representación de la mujer en la ficción literaria? El tema del doble ofrece un campo de exploración siempre abierto a variar la respuesta a estas preguntas, a comprobar si la búsqueda de una voz imparcial en lo femenino es ya posible, o si, por el contrario, tal aspiración se reveló agotada desde el principio al grito de «I am her!».

\section{BiBLIOGRAFÍA}

Abel, Elizabeth (1982): Writing and sexual difference, University of Chicago Press, Chicago.

Alpini, Gloria (2009): The Female Fantastic: Evolution, Theories and the Poetics of Perversion, Aras Edizioni, Fano.

Anodos, [pseud. de Mary E. Coleridge] (1896): Fancy's Following, Daniel Press, Oxford. BARGALló, Juan (1994): «Hacia una tipología del doble: el doble por fusión, por fisión y por metamorfosis», en Juan Bargalló (ed.), Identidad y alteridad, aproximación al tema del «doble», Alfar, Sevilla, pp. 11-26.

BRAun, Heather (2016): «Mary E. Coleridge, Androgyny, and The Spectral Doppelgänger», Parlour, núm. 2, disponible en <https://www.ohio.edu/cas/parlour/ news / library/spectral-doppelganger $>$ [fecha de consulta: 1 de mayo de 2020]

Bremond, Claude (2003): «Concepto y Tema», en Cristina Naupert (ed.), Tematología y comparatismo literario, Arco/Libros, Madrid, pp. 167-180.

ButLer, Judith (1990): Gender Trouble: Feminism and the Subversion of Identity, Routledge, Nueva York.

CAillors, Roger (1965): Au coeur du fantastique, Gallimard, París.

CanetTi, Elias (1960): Masse und Macht, Claasen, Hamburgo.

CoAtes, Paul (1988): The Double and The Other: Identity as Ideology in Post-Romantic Fiction, Macmillan, Londres.

CORTÁZAR, Julio (1982): Bestiario, Alfaguara, Madrid.

Delashmit, Margaret (1990): The Patriarchy and Women: A Study of Charlotte Perkins Gilman's «The Yellow Wallpaper», University of Tennessee-Knoxville, Knoxville.

Dock, Julie Bates (1998): Charlotte Perkins Gilman's the Yellow Wall-Paper and the History of Its Publication and Reception: A Critical Edition and Documentary Casebook, The Pennsylvania State University Press, State College, PA.

DoležEL, Lubomír (2003): «Una semántica para la temática: el caso del doble», en Cristina Naupert (ed.), Tematología y comparatismo literario, Arco/Libros, Madrid, pp. 257-275. 
Fernández CubAs, Cristina (1988): Mi hermana Elba / Los altillos de Brumal, Tusquets, Barcelona.

Freud, Sigmund (1957): The Complete Works of Sigmund Freud, ed. James Strachey, Hogarth, Londres.

Fusillo, Massimo (1998): L'altro e lo stesso, La Nuova Italia, Florencia.

GILBERT, Sandra, y Susan Gubar (1979): The Madwoman in the Attic: The Woman Writer and the Nineteenth-Century Literary Imagination, Yale University Press, New Haven.

Gilman, Charlotte Perkins (1910): The Home. Its Work and Influence, The Co-Operative Press, Nueva York.

— (1913): «Why I Wrote "The Yellow Wallpaper"?», The Forerunner, pp. 19-20.

- (1973): The Yellow Wallpaper, Virago Press, Londres.

- (1990): The Living of Charlotte Perkins Gilman, An Autobiography, ed. Ann J. Lane, The University of Wisconsin Press, Madison.

GreEne, Gayle y Coppélia KaHN (eds.) (1985): Making a Difference: Feminist Literary Criticism, Methuen, Londres.

Hedges, Elaine R. (1988): «Afterword», en Charlotte Perkins Gilman, The Yellow Wallpaper, Virago Press, Londres, pp. 37-63.

Jourde, Pierre, y Paolo Tortonese (1996): Visages du double: un thème littéraire, Nathan, París.

Lauretis, Teresa de (1992): Alicia ya no: feminismo, semiótica, cine, Cátedra, Valencia.

Llorente, María Ema (2019): «Literatura fantástica y discurso lírico. Caracterización y análisis de la poesía fantástica española actual», Tropelías, núm. 31, pp. 297-320.

Martín, Rebeca (2006): Las manifestaciones del doble en la narrativa breve española contemporánea, Universitat Autònoma de Barcelona, Bellaterra. [Tesis doctoral]

McGowan, Todd (2001): The Feminine «No!», State University of New York Press, Nueva York.

PoE, Edgar Allan (2009): Complete Stories, Douglas, Vancouver.

Poelen, Anne (2005): «Esquizofrenia en "Lúnula y Violeta”. El crecimiento caprichoso del doble», Lectora, núm. 11, pp. 235-247.

QuAWAS, Rula (2006): «A New Woman's Journey into Insanity: Descent and Return in "The Yellow Wallpaper"», Journal of the Australasian Universities Language and Literature Assotiation, núm. 105, pp. 35-53.

Rank, Otto (1971): The Double: A Psychoanalytic Study, trad. Harry Tucker Jr., The University of North Carolina Press, Chapel Hill.

Santana, Rita (2018): «Que Papel de Parede? Uma Leitura Do Conto “O Papel de Parede Amarelo" de Charlotte Perkins Gilman», Faces de Eva. Estudos Sobre a Mulher, núm. 39, pp. 93-109.

Todorov, Tzvetan (1994): Introducción a la literatura fantástica, trad. Silvia Delpuy, Coyoacán, México.

Vilella, Eduard (1999): El doble: elements per a una panorámica històrica, Publicacions Universitat de Barcelona, Barcelona.

WebBer, Andrew J. (1996): The Doppelgänger: Double Visions in German Literature, Oxford University Press, Oxford. 